\title{
Optimal estimator for the amplitude of the bispectrum from infrared clustered sources
}

\author{
F. Lacasa and N. Aghanim \\ Institut d'Astrophysique Spatiale (IAS), Bâtiment 121, Université Paris-Sud 11 and CNRS (UMR 8617), 91405 Orsay, France \\ e-mail: [Fabien.Lacasa;Nabila.Aghanim]@ias.u-psud.fr
}

Received 16 November 2012 / Accepted 25 June 2014

\section{ABSTRACT}

\begin{abstract}
We devise a fast and optimal estimator for the amplitude of the bispectrum of the clustered point sources constituting the cosmic infrared background (CIB). We show how this estimator can account for the cases of partial sky coverage and inhomogeneous noise. The expected detection significance is presented in terms of signal-to-noise, showing that the CIB bispectrum will be undetectable below $220 \mathrm{GHz}$ with a Planck-like experiment; in contrast, detection may be achieved at or above $220 \mathrm{GHz}$, especially if the CMB is removed. We also show how this estimator can be combined with estimators of unclustered sources and CMB non-Gaussianity to build up joint robust constraints. On the one hand, we find that, for a Planck-like experiment, CMB non-Gaussianity estimation can be decoupled from point source contributions, if the brightest sources are masked. On the other hand, we find that the estimation of clustered and unclustered sources non-Gaussianity are strongly coupled, which diminishes their separate detection significance.
\end{abstract}

Key words. galaxies: star formation - cosmic background radiation - cosmology: observations - large-scale structure of Universe submillimeter: diffuse background

\section{Introduction}

The measurement and study of high-order moments of the cosmic microwave background (CMB) and the large scale structure (LSS) has emerged as an important field in observational cosmology because it probes deviations from Gaussianity. For example, observing non-Gaussianity (NG) from the CMB or the large scale structure would probe primordial NG dating back to the generation of cosmological perturbations, and this in turn would constrain severely the primordial models generating these fluctuations (e.g. inflation). For example, a NG detection with the sensitivities of today's experiments would rule out a whole class of inflation models, among which the standard single-field slow roll inflation (Acquaviva et al. 2003; Maldacena 2003; Creminelli \& Zaldarriaga 2004). However, the latest measurement by the Planck Collaboration has put stringent constraints on this possibility (Planck Collaboration XXIV 2014).

The most popular form for this primordial non-Gaussianity is the so-called "local" type, parametrised by a factor $f_{\mathrm{NL}}$, for which the Bardeen potential takes the form

$\Phi(\boldsymbol{x})=\Phi_{\mathrm{G}}(\boldsymbol{x})+f_{\mathrm{NL}}\left(\Phi_{\mathrm{G}}(\boldsymbol{x})^{2}-\left\langle\Phi_{\mathrm{G}}(\boldsymbol{x})^{2}\right\rangle\right)$,

where $\Phi_{\mathrm{G}}$ is the Gaussian part of the potential. It is on this form of NG that most efforts have focused and that the tightest constraints were obtained. Indeed a fast estimators for $f_{\mathrm{NL}}$ was developed and applied to the CMB data (Komatsu et al. 2005, 2009, 2011; Planck Collaboration XXIV 2014). However, at $\mathrm{CMB}$ frequencies, many contaminating signals are present, which typically have a non-Gaussian distribution. While the galactic emission is mainly confined to a defined area, the galactic plane, and may thus be masked, extragalactic point sources are present all over the sky. The brightest sources can be masked but the faintest, below detection limits and confusion levels, can neither be detected nor masked. These unresolved sources are therefore one potential source of secondary non-Gaussianity that needs to be estimated.

Essentially, at $\mathrm{CMB}$ frequencies the extragalactic point sources split into two main populations defined with respect to the characteristic frequency domain they emit at, namely radio and infrared (IR). We thus consider radio loud sources with strong magnetic fields that yield synchrotron or free-free emission in the radio domain and IR galaxies with thermal emission from dust heated by young stars in the IR domain.

At the CMB frequencies, radio galaxies can be considered as randomly distributed on the sky (Toffolatti et al. 1998; González-Nuevo et al. 2005). They thus have constant polyspectra, in particular their power spectrum and bispectrum. They are hence characterised entirely by their number counts, with, say, the latest model by Tucci et al. (2011). In this context, their bispectrum can be written as

$b_{\mathrm{PS}}=\int S^{3} \frac{\mathrm{d} n}{\mathrm{~d} S} \mathrm{~d} S$,

where $S$ is the source flux density in Jy. ( $b_{\mathrm{PS}}$ may then be converted to CMB temperature elevation through Planck's law, but is usually quoted in dimensionless units $\Delta T / T$ in the literature).

Unlike radio galaxies, the cosmic infrared background (CIB), produced by IR galaxies, is strongly clustered. Not only are galaxy number counts important for modelling this case, but also the distribution of the CIB anisotropies. Models for the IR galaxies number counts (Lapi et al. 2011; Somerville et al. 2012; Béthermin et al. 2012) and their power spectrum (Pénin et al. 2012; Xia et al. 2012; Thacker et al. 2013) have recently been developed. Furthermore, the most recent CMB experiments, such as Planck (Planck Collaboration I 2014; Planck Collaboration I 2011), the Atacama Cosmology Telescope (Das et al. 2011) and the South Pole Telescope (Keisler et al. 2011), that are probing high frequencies have permitted the detection of CIB on several 
scales (e.g. Hall et al. 2010; Planck Collaboration XVIII 2011; Viero et al. 2013; Planck Collaboration XXX 2014). At present, extragalactic point sources are thus well characterised at the 1and 2-point levels, but the study of their higher orders is still in its early stages. Theoretical predictions were performed by Arguieso et al. (2003) and Lacasa et al. (2012), among others. The estimate of contamination of the primordial NG parameter $f_{\mathrm{NL}}$ by the point sources was performed for example by Babich \& Pierpaoli (2008), Lacasa et al. (2012) in temperature, and by Curto et al. (2013) accounting for polarisation. They show that radio and IR populations yield different biases since their bispectra have different shapes. Statistical methods of estimating the non-Gaussianity from radio point sources, based on wavelets (Argüeso et al. 2006) or on the bispectrum (Komatsu et al. 2005), have been developed, and first measurements were made on WMAP data (Komatsu et al. 2009, 2011). Recently, detection of the bispectrum of the CIB anisotropies was performed by Planck Collaboration XXX (2014) and Crawford et al. (2014).

The purpose of this article is to propose a fast estimator, taking advantage of a prescription developed in Lacasa et al. (2012) for measuring the amplitude of the non-Gaussianity (bispectrum) of clustered point sources. This estimator can be used in non-Gaussianity studies to disentangle the primordial and foreground contributions, and among foregrounds it can be used to disentangle the contributions from clustered and unclustered point sources, which may help for putting constraints on their respective models.

The article is organised as follows. In Sect. 2 we briefly recall the prescription for the bispectrum of IR sources and introduce the estimator for its amplitude. In Sect. 3 we tackle the problems of partial sky coverage and anisotropic noise. In Sect. 4 we present the expected signal-to-noise ratio $(\mathrm{S} / \mathrm{N})$ of detection at several frequencies. In Sect. 5 we show how to combine our new estimator with the ones used for the $\mathrm{CMB}$ and radio galaxies to produce a joint constraint of non-Gaussianity, and we conclude in Sect. 6.

\section{Bispectrum of clustered sources}

\subsection{Bispectrum}

Given a full-sky map of the temperature fluctuations $\Delta T(n)$ of some signal in direction $\boldsymbol{n}$, it can be decomposed in the spherical harmonic basis

$a_{\ell m}=\int \mathrm{d}^{2} \boldsymbol{n} Y_{\ell m}^{*}(\boldsymbol{n}) \Delta T(\boldsymbol{n})$

with the usual orthonormal spherical harmonics $Y_{\ell m}$

$\int \mathrm{d}^{2} \boldsymbol{n} Y_{\ell m}(\boldsymbol{n}) Y_{\ell^{\prime} m^{\prime}}^{*}(\boldsymbol{n})=\delta_{\ell \ell^{\prime}} \delta_{m m^{\prime}}$.

The power spectrum $C_{\ell}$ of the signal is the 2-point correlation function in harmonic space given as

$\left\langle a_{\ell m} a_{\ell^{\prime} m^{\prime}}^{*}\right\rangle=C_{\ell} \delta_{\ell \ell^{\prime}} \delta_{m m^{\prime}}$.

The bispectrum $b_{\ell_{1} \ell_{2} \ell_{3}}$ is the three-point correlation function in harmonic space:

$\left\langle a_{\ell_{1} m_{1}} a_{\ell_{2} m_{2}} a_{\ell_{3} m_{3}}\right\rangle=G_{\ell_{1} \ell_{2} \ell_{3}}^{m_{1} m_{2} m_{3}} \times b_{\ell_{1} \ell_{2} \ell_{3}}$

with the Gaunt coefficient

$$
\begin{aligned}
G_{1,2,3} & =\int \mathrm{d}^{2} \boldsymbol{n} Y_{123}(\boldsymbol{n}) \\
& =\sqrt{\frac{(2 \ell+1)_{123}}{4 \pi}}\left(\begin{array}{ccc}
\ell_{1} & \ell_{2} & \ell_{3} \\
0 & 0 & 0
\end{array}\right)\left(\begin{array}{ccc}
\ell_{1} & \ell_{2} & \ell_{3} \\
m_{1} & m_{2} & m_{3}
\end{array}\right)
\end{aligned}
$$

where $Y_{i}=Y_{\ell_{i} m_{i}}$. In the following the subscript 123 denotes the product of the corresponding variables e.g. $X_{123} \equiv X_{1} X_{2} X_{3}$. $G_{1,2,3}$ is zero unless the triplet $\left(\ell_{1}, \ell_{2}, \ell_{3}\right)$ follows the triangle inequalities and $m_{1}+m_{2}+m_{3}=0$.

The bispectrum estimator is

$$
\hat{b}_{123}=\frac{1}{N_{123}} \sum_{m_{1}, m_{2}, m_{3}} G_{1,2,3} a_{1} a_{2} a_{3}
$$

with

$N_{\ell_{1} \ell_{2} \ell_{3}}=\frac{(2 \ell+1)_{123}}{4 \pi}\left(\begin{array}{ccc}\ell_{1} & \ell_{2} & \ell_{3} \\ 0 & 0 & 0\end{array}\right)^{2}$

being the "number of modes" for the $\left(\ell_{1}, \ell_{2}, \ell_{3}\right)$ triangle.

In the following, we note in bold the bispectrum that accounts for the beam effect $B_{\ell}$

$\boldsymbol{b}_{1,2,3}=b_{\ell_{1} \ell_{2} \ell_{3}} B_{\ell_{1}} B_{\ell_{2}} B_{\ell_{3}}$.

We also note that

$C_{\ell}=C_{\ell} B_{\ell}^{2}+C_{\ell}^{\text {noise }}$

is the power spectrum accounting for both the noise and the beam effects.

\subsection{Optimal estimator of the amplitude of the bispectrum}

The non-Gaussianity from unclustered sources at CMB frequencies is entirely characterised by a single parameter $b_{\mathrm{PS}}$ that can be estimated on a map (Komatsu et al. 2005). As a result their contamination to $f_{\mathrm{NL}}$ can be marginalised over. By contrast, the non-Gaussianity from clustered sources is more complicated and is characterised by the full bispectrum, whose estimation is computationally intensive (with $O\left(N_{\text {pix }}^{5 / 2}\right)$ operations).

In this context and following work by Argüeso et al. (2003), Lacasa et al. (2012) proposed a full-sky analytical prescription to compute the bispectrum of extragalactic sources from their number counts and power spectra. In this prescription, the bispectrum arising from a general population of point sources writes as

$b_{\ell_{1} \ell_{2} \ell_{3}}^{\mathrm{PS}}=\alpha \sqrt{C_{\ell_{1}} C_{\ell_{2}} C_{\ell_{3}}}$

with $C_{\ell}$ being the power spectrum of the considered point sources and

$\alpha=\frac{\int S^{3} \frac{\mathrm{d} N}{\mathrm{~d} S} \mathrm{~d} S}{\left(\int S^{2} \frac{\mathrm{d} N}{\mathrm{~d} S} \mathrm{~d} S\right)^{3 / 2}}=\frac{b_{1,2,3}^{\text {white }}}{\left(C_{\ell}^{\text {white }}\right)^{3 / 2}}$

where $S$ is the source flux density. The integral runs up to the detection limit of the resolved sources indicated by a flux cut $S_{\text {cut }}, \frac{\mathrm{d} N}{\mathrm{~d} S}$ are the number counts per steradian, and the superscript "white" denotes the contribution from a white-noise with the same number counts. Equation (11) reduces to the usual formula $b_{\mathrm{PS}}=\int S^{3} \frac{\mathrm{d} N}{\mathrm{~d} S} \mathrm{~d} S$ in the unclustered case $\left(C_{\ell}=C_{\ell}^{\text {white }}\right)$. In that sense Eq. (11) is a generalisation that includes the clustering effect.

Lacasa et al. (2012) have tested the validity of the prescription against numerical simulations developed by Sehgal et al. (2010). They have shown that the prescription agrees with the full bispectrum measured on the simulated maps.

The level of non-Gaussianity induced by point sources can be estimated by measuring the bispectrum directly, or it can be 
quantified by the amplitude of their bispectra compared to a template bispectrum. Such an estimator has already been proposed for the unclustered sources (Komatsu et al. 2005). In the following, we construct a similar estimator of the bispectrum amplitude for the clustered sources. To do so, we define the template bispectrum as the prescription given in Eq. (11). In practice, the amplitude of the bispectrum is measured by minimising the $\chi^{2}$ of the map bispectrum to the template:

$\chi^{2}(A)=\sum_{\ell_{1} \leq \ell_{2} \leq \ell_{3}} \frac{\left(\boldsymbol{b}_{1,2,3}-A \boldsymbol{b}_{1,2,3}^{\text {clust }}\right)^{2}}{\sigma^{2}\left(\ell_{1}, \ell_{2}, \ell_{3}\right)}$

with

$\sigma^{2}\left(\ell_{1}, \ell_{2}, \ell_{3}\right)=\frac{C_{\ell_{1}}^{\text {tot }} C_{\ell_{2}}^{\text {tot }} C_{\ell_{3}}^{\text {tot }}}{N_{\ell_{1} \ell_{2} \ell_{3}}} \times \Delta_{\ell_{1} \ell_{2} \ell_{3}}$

being the bispectrum variance in the weak non-Gaussianity limit $^{1}$. In this expression, $C_{\ell}^{\text {tot }}$ is the map power spectrum, and we have

$$
\begin{aligned}
\Delta_{\ell_{1} \ell_{2} \ell_{3}} & =1+\delta_{\ell_{1} \ell_{2}}+\delta_{\ell_{1} \ell_{3}}+\delta_{\ell_{2} \ell_{3}}+2 \delta_{\ell_{1} \ell_{2} \ell_{3}} \\
& =\left\{\begin{array}{l}
6 \text { for equilateral triangle } \\
2 \text { for isosceles triangle } \\
1 \text { for general triangle }
\end{array}\right.
\end{aligned}
$$

Then the maximum likelihood estimator is

$\hat{A}=\sum_{\ell_{1} \leq \ell_{2} \leq \ell_{3}} \frac{\boldsymbol{b}_{1,2,3} \boldsymbol{b}_{1,2,3}^{\text {clust }}}{\sigma^{2}\left(\ell_{1}, \ell_{2}, \ell_{3}\right)} \times \sigma^{2}(\hat{A})$

with

$\sigma^{2}(\hat{A})=\left(\sum_{\ell_{1} \leq \ell_{2} \leq \ell_{3}} \frac{\left(\boldsymbol{b}_{1,2,3}^{\text {clust }}\right)^{2}}{\sigma^{2}\left(\ell_{1}, \ell_{2}, \ell_{3}\right)}\right)^{-1}$

being a normalisation factor giving the error bar in the assumption of a Gaussian likelihood, which is valid in the weak NG limit when $\sigma^{2}\left(\ell_{1}, \ell_{2}, \ell_{3}\right)$ can be considered independent of $A$.

The theoretical bispectrum, Eq. (11), is separable ${ }^{2}$; hence a simpler computation of the numerator of Eq. (16) can be devised, inspired by Komatsu et al. (2005). Indeed, defining the filtered map,

$F(\boldsymbol{n})=\sum_{\ell m} \frac{\alpha^{1 / 3} \sqrt{C_{\ell}^{\text {clust }}} b_{\ell}}{C_{\ell}^{\text {tot }}} a_{\ell m} Y_{\ell m}(\boldsymbol{n})$

and

$\mathcal{S}_{\text {clust }}=\int \mathrm{d}^{2} \boldsymbol{n} F(\boldsymbol{n})^{3}$,

we have

$\mathcal{S}_{\text {clust }}=\sum_{\ell_{1} \ell_{2} \ell_{3}} \frac{\boldsymbol{b}_{1,2,3}^{\text {clust }} \hat{\boldsymbol{b}}_{123}}{C_{\ell_{1}}^{\text {tot }} C_{\ell_{2}}^{\text {tot }} C_{\ell_{3}}^{\text {tot }}} N_{123}=6 \times \sum_{\ell_{1} \leq \ell_{2} \leq \ell_{3}} \frac{\boldsymbol{b}_{1,2,3}^{\text {clust }} \hat{\boldsymbol{b}}_{123}}{\sigma^{2}\left(\ell_{1}, \ell_{2}, \ell_{3}\right)}$.

Furthermore, it is worth noting that this approach is faster than a full bispectrum analysis, with $O\left(N_{\text {pix }}^{3 / 2}\right)$ operations.

\footnotetext{
1 This approximation is justified for the CIB, which is nearly Gaussian (see Lacasa et al. 2012 for details), or when the maps are contaminated by the CMB.

2 A bispectrum is said to be separable if it can be written in the form $b\left(\ell_{1}, \ell_{2}, \ell_{3}\right)=\sum_{i} f_{i}\left(\ell_{1}\right) g_{i}\left(\ell_{2}\right) h_{i}\left(\ell_{3}\right)+$ perm. This is the case for the CIB bispectrum with the sum reducing to one element and $f_{i}(\ell)=g_{i}(\ell)=$ $h_{i}(\ell)=\alpha^{1 / 3} \sqrt{C_{\ell}^{\mathrm{IR}}}$.
}

\section{Masked sky and inhomogeneous noise}

In realistic cases of $\mathrm{CMB}$ analysis, the statistical isotropy of the signal is broken by inhomogeneous noise, e.g. due to scanning strategy, or by masking large areas of the sky, e.g. those contaminated by galactic emissions. In these cases, the estimator of the bispectrum amplitude for clustered sources is no longer optimal and is biased in a non-trivial way. Nevertheless and similar to the case of $f_{\mathrm{NL}}$, the bias and the lack of optimality can both be tackled through adapted modifications to the estimator.

\subsection{Debiasing}

When a covariance matrix of the map(s) can be estimated, Creminelli et al. (2006) have shown that Wiener filtering of the map(s) will debias the non-Gaussian estimator from anisotropic contaminants or noise. Specifically, if $\mathbf{C}$ is the estimated covariance matrix in harmonic space, the estimator Eq. (16) can be debiased by applying the change

$\frac{a_{\ell m}}{C_{\ell}^{\mathrm{tot}}} \rightarrow a_{\ell m}^{\prime}=\left(\mathbf{C}^{-1} \cdot \boldsymbol{a}\right)_{\ell m}=\sum_{\ell^{\prime} m^{\prime}} C_{\ell m, \ell^{\prime} m^{\prime}}^{-1} a_{\ell^{\prime} m^{\prime}}$

in the filtered map Eq. (18), where $\boldsymbol{a}=\left(a_{\ell m}\right)_{\ell=2 . . \ell_{\max }, m=-\ell . . \ell}$ is the vector of harmonic coefficients.

This step is sometimes called $\mathbf{C}^{-1}$ prefiltering, and an efficient algorithm for it has been designed by Elsner \& Wandelt (2013).

\subsection{Reducing variance and reaching optimality}

When isotropy is broken, the three-point correlation function used to define the bispectrum in Eq. (6) no longer has minimal variance. It must be replaced by the Wick product of the three harmonic coefficients given by

$$
\begin{aligned}
a_{\ell_{1} m_{1}} a_{\ell_{2} m_{2}} a_{\ell_{3} m_{3}} \rightarrow & a_{\ell_{1} m_{1}} a_{\ell_{2} m_{2}} a_{\ell_{3} m_{3}}-\left\langle a_{\ell_{1} m_{1}} a_{\ell_{2} m_{2}}\right\rangle a_{\ell_{3} m_{3}} \\
& -\left\langle a_{\ell_{1} m_{1}} a_{\ell_{3} m_{3}}\right\rangle a_{\ell_{2} m_{2}}-\left\langle a_{\ell_{2} m_{2}} a_{\ell_{3} m_{3}}\right\rangle a_{\ell_{1} m_{1}}
\end{aligned}
$$

This expression exhibits the same mean as in the isotropic case but it has a lower variance. Furthermore, when applied to the isotropic case Eq. (22) gives the same bispectrum estimator as in Eq. (6) (e.g. for $\ell_{1} \neq \ell_{2} \neq \ell_{3}$ all the expectation values vanish).

Only the two-point correlation function is to be considered for the expectation values. Therefore in practice, the latter are obtained from a sufficiently large number of Gaussian realisations with same power spectrum as that of the signal considered.

Inputting the linear corrections given by Eq. (22) into the expression of $S_{\mathrm{IR}}$ in Eq. (20) yields

$$
S_{\text {clust }} \rightarrow \tilde{S}_{\text {clust }}=\int \mathrm{d}^{2} \boldsymbol{n} F(\boldsymbol{n})^{3}-3 \times \int \mathrm{d}^{2} \boldsymbol{n} F(\boldsymbol{n}) G(\boldsymbol{n})
$$

with

$$
\begin{aligned}
G(\boldsymbol{n}) & =\sum_{12} \alpha^{2 / 3} \sqrt{C_{\ell_{1}}^{\mathrm{IR}} C_{\ell_{2}}^{\mathrm{IR}}}\left\langle a_{1}^{\prime} a_{2}^{\prime}\right\rangle_{\mathrm{MC}} Y_{1}(\boldsymbol{n}) Y_{2}(\boldsymbol{n}) \\
& =\left\langle F(\boldsymbol{n})^{2}\right\rangle_{\mathrm{MC}}
\end{aligned}
$$

where the brackets \langle\rangle$_{\mathrm{MC}}$ stand for the average of Gaussian simulations. 


\section{Expected detection significance for the CIB}

We now apply our formalism for clustered sources to the case of the CIB. We consider here both lensed and unlensed IR galaxies as a single population of dusty star-forming galaxies.

We present the expected detection significance in terms of the $\mathrm{S} / \mathrm{N}$ for the CIB bispectrum amplitude estimator. To this end, we make use of the values of $\alpha$ and $C_{\ell}^{\mathrm{CIB}}$ that we measure on the simulations by Sehgal et al. (2010). These publicly available simulations developed for the Atacama Cosmology Telescope experiment cover, in particular, the frequencies 150, 220, 280 and $350 \mathrm{GHz}$. Furthermore, these values of $\alpha$ and $C_{\ell}^{\mathrm{CIB}}$ were shown to adequately reproduce the CIB bispectrum from 150 to $350 \mathrm{GHz}$ frequencies (Lacasa et al. 2012). The instrumental characteristics (beams and noise levels) used are specified in Table 2.

\subsection{Ideal cosmic variance-limited case}

We first focus on an ideal case where the signal is made solely of CIB galaxies, i.e. noise and other astrophysical contributions are neglected. We will compute the $\mathrm{S} / \mathrm{N}$ of the detection of the nonGaussian signal as defined by the amplitude of the bispectrum. Each triplet $\ell_{1} \leq \ell_{2} \leq \ell_{3}$ contains information proportional to its number of configurations:

$\sigma^{-2}(\hat{A})=\alpha^{2} \sum_{\ell_{1} \leq \ell_{2} \leq \ell_{3}} N_{\ell_{1} \ell_{2} \ell_{3}}$.

In other terms, the $\mathrm{S} / \mathrm{N}$ of the detection increases with the total number of bispectrum configurations:

$S / N=\frac{1}{\sigma(\hat{A})} \propto \sqrt{N_{\text {tot }}\left(\ell_{\max }\right)}$

with

$N_{\text {tot }}\left(\ell_{\max }\right)=\sum_{\ell_{\min } \leq \ell_{1} \leq \ell_{2} \leq \ell_{3} \leq \ell_{\max }} N_{\ell_{1} \ell_{2} \ell_{3}}$.

For example in the case of full-sky maps of the CIB at frequencies $150,220,280$, and $350 \mathrm{GHz}$ with angular scales ranging from $\ell_{\min }=2$ to $\ell_{\max }=2048$, the obtained $\mathrm{S} / \mathrm{N}$ are $\sim 1000$, and they do not vary much from frequency to frequency (see first row of Table 1).

\subsection{Realistic case $(C M B+C I B)$}

In a real experiment, the CIB map is convolved by the instrumental beam, and the signal is contaminated by noise and other astrophysical/cosmological signals. The most prominent contaminants are the CMB and the galaxy, where the latter can be masked. The brightest point sources are also masked. Hence the $\mathrm{S} / \mathrm{N}$ is significantly decreased compared to the ideal case. Table 1 summarises the expected $\mathrm{S} / \mathrm{N}$ with the instrumental characteristics specified in Table 2. We show how the detection significance decreases from the ideal case to the case of a convolved but "perfect" full-sky CIB map, then including CMB contamination but still full-sky, and finally with a 50\% sky-fraction mimicking the mask that may be applied to avoid galactic dust emission. We also include the case where $90 \%$ of the CMB can be removed by component separation methods. As a matter of fact, component separation methods can efficiently estimate the CMB signal from multifrequency observation with an error in amplitude below 10\% (see Delabrouille \& Cardoso 2007; Remazeilles et al. 2011; Bobin et al. 2013 and references therein). The estimated CMB map can then be substracted from frequency maps
Table 1. Expected $\mathrm{S} / \mathrm{N}$ at $\ell_{\max }=2048$.

\begin{tabular}{ccccc}
\hline \hline Frequency $(\mathrm{GHz})$ & 150 & 220 & 280 & 350 \\
\hline Ideal case & 1218 & 1157 & 1161 & 1159 \\
Full sky CIB & 15.5 & 98 & 336 & 833 \\
Full sky with CMB & 0.39 & 6.7 & 55 & 387 \\
50\% sky with CMB & 0.28 & 4.7 & 39 & 274 \\
50\% sky with 10\% CMB & 3.67 & 45.9 & 210 & 577 \\
\hline
\end{tabular}

Notes. Ideal stands for a full-sky cosmic-variance limited CIB map without noise or contaminations. All subsequent rows adopt the instrumental characteristics specified in Table 2. Full sky CIB represents the case of the CIB map as seen by the instrumental set-up. Full sky with $\mathrm{CMB}$ is when CMB is present, contaminating the CIB map. 50\% sky with $\mathrm{CMB}$ stands for the case of masking half of the sky. $50 \%$ sky with $10 \%$ CMB stands for a half sky CIB map where $90 \%$ of the CMB (in amplitude) has been removed by some component separation.

Table 2. Instrumental specifications used throughout this article.

\begin{tabular}{ccc}
\hline \hline Beam & Noise & $C_{\ell}^{\text {noise }}$ \\
\hline 5 arcmin & $10^{-8} \Delta T / T \times \mathrm{sr}^{1 / 2}$ & $7.4 \times 10^{-4} \mu \mathrm{K}^{2} \times \mathrm{sr}$ \\
\hline
\end{tabular}

to reveal the weaker CIB signal from unresolved sources as performed for example by Planck Collaboration XVIII (2011) and Planck Collaboration XXX (2014). For the partial-sky coverage cases, we assume that the optimisation and the debiasing described in Sects. 3.1 and 3.2 have been applied with the $\mathrm{S} / \mathrm{N}$ scaling as $f_{\mathrm{SKY}}^{-1 / 2}$.

The $\mathrm{S} / \mathrm{N}$ of the detection is severely reduced from ideal to more realistic cases especially at lower frequencies. The signal is still detectable at all frequencies when including beam and noise effects. However, when the $\mathrm{CMB}$ is included the signal becomes undetectable at $150 \mathrm{GHz}$. Above this frequency the detection significance is $S / N \sim 5 \sigma$ at $220 \mathrm{GHz}$ and reaches 40 to $300 \sigma$ at 280 and $350 \mathrm{GHz}$ where the CIB emission is dominant even in presence of CMB contribution.

The removal of the CMB component from the frequency maps allows us to further improve the detection at $220 \mathrm{GHz}$ by increasing the $\mathrm{S} / \mathrm{N}$ to $\sim 46 \sigma$. In this case, the CIB nonGaussianity at $150 \mathrm{GHz}$ seems marginally detectable. However at this frequency the non-Gaussianity from radio sources becomes a problem (while at higher frequencies IR sources are forecasted to dominate the unresolved source population).

\section{Joint NG estimation}

When several non-Gaussian signals are present, a joint estimation of their amplitudes that takes their covariances into account is necessary. In the present study we focus on the main extragalactic non-Gaussian signals: unclustered sources (in particular radio sources, but also IR late-type galaxies and, more generally, any population exhibiting constant polyspectra), the CIB, and the $\mathrm{CMB}$, with potential local-type non-Gaussianity. A joint estimation requires minimisation of the chi-square:

$\chi^{2}\left(f_{\mathrm{NL}}, b_{\mathrm{PS}}, A_{\mathrm{CIB}}\right)=\sum_{\ell_{1} \leq \ell_{2} \leq \ell_{3}} \frac{\left(\boldsymbol{b}_{1,2,3}^{\mathrm{obs}}-\boldsymbol{b}_{1,2,3}^{\text {model }}\left(f_{\mathrm{NL}}, b_{\mathrm{PS}}, A_{\mathrm{CIB}}\right)\right)^{2}}{\sigma^{2}\left(\ell_{1}, \ell_{2}, \ell_{3}\right)}$

with

$\boldsymbol{b}_{1,2,3}^{\text {model }}\left(f_{\mathrm{NL}}, b_{\mathrm{PS}}, A_{\mathrm{CIB}}\right)=f_{\mathrm{NL}} \boldsymbol{b}_{1,2,3}^{\mathrm{CMB}}+b_{\mathrm{PS}} \boldsymbol{b}_{1,2,3}^{\text {unclust }}+A_{\mathrm{CIB}} \boldsymbol{b}_{1,2,3}^{\mathrm{CIB}}$ 
and (involved in the definition of $\sigma^{2}\left(\ell_{1}, \ell_{2}, \ell_{3}\right)$, see Eq. (14))

$C_{\ell}^{\text {tot }}=\left(C_{\ell}^{\mathrm{CMB}}+C_{\ell}^{\mathrm{unclust}}+C_{\ell}^{\mathrm{CIB}}\right) B_{\ell}^{2}+C_{\ell}^{\text {noise }}$.

We use the standard parameter $b_{\mathrm{PS}}$ for compatibility with the literature (instead of noting $A_{\text {unclust }}$ ).

Then, if we define the scalar product between two bispec$\operatorname{tra} \boldsymbol{b}^{\alpha}$ and $\boldsymbol{b}^{\beta}$ :

$$
\left\langle\boldsymbol{b}^{\alpha}, \boldsymbol{b}^{\beta}\right\rangle=\sum_{\ell_{1} \leq \ell_{2} \leq \ell_{3}} \frac{\boldsymbol{b}_{1,2,3}^{\alpha} \boldsymbol{b}_{1,2,3}^{\beta}}{\sigma^{2}\left(\ell_{1}, \ell_{2}, \ell_{3}\right)} .
$$

Minimising Eq. (29) corresponds to solving the linear system:

$$
\begin{gathered}
\left(\begin{array}{ccc}
\left\langle\boldsymbol{b}_{\mathrm{CMB}}, \boldsymbol{b}_{\mathrm{CMB}}\right\rangle & \left\langle\boldsymbol{b}_{\mathrm{uncl}}, \boldsymbol{b}_{\mathrm{CMB}}\right\rangle & \left\langle\boldsymbol{b}_{\mathrm{CIB}}, \boldsymbol{b}_{\mathrm{CMB}}\right\rangle \\
\left\langle\boldsymbol{b}_{\mathrm{CMB}}, \boldsymbol{b}_{\mathrm{uncl}}\right\rangle & \left\langle\boldsymbol{b}_{\mathrm{uncl}}, \boldsymbol{b}_{\mathrm{uncl}}\right\rangle & \left\langle\boldsymbol{b}_{\mathrm{CIB}}, \boldsymbol{b}_{\mathrm{uncl}}\right\rangle \\
\left\langle\boldsymbol{b}_{\mathrm{CMB}}, \boldsymbol{b}_{\mathrm{CIB}}\right\rangle & \left\langle\boldsymbol{b}_{\mathrm{uncl}}, \boldsymbol{b}_{\mathrm{CIB}}\right\rangle & \left\langle\boldsymbol{b}_{\mathrm{CIB}}, \boldsymbol{b}_{\mathrm{CIB}}\right\rangle
\end{array}\right) \cdot\left(\begin{array}{c}
f_{\mathrm{NL}} \\
b_{\mathrm{PS}} \\
A_{\mathrm{CIB}}
\end{array}\right)= \\
\left(\begin{array}{c}
\left\langle\boldsymbol{b}_{\mathrm{obs}}, \boldsymbol{b}_{\mathrm{CMB}}\right\rangle \\
\left\langle\boldsymbol{b}_{\mathrm{obs}}, \boldsymbol{b}_{\mathrm{uncl}}\right\rangle \\
\left\langle\boldsymbol{b}_{\mathrm{obs}}, \boldsymbol{b}_{\mathrm{CIB}}\right\rangle
\end{array}\right) .
\end{gathered}
$$

If we introduce-noted with upper tilde-the estimators which consider only one source of non-Gaussianity, e.g.

$\tilde{A}_{\mathrm{CIB}}=\frac{\left\langle\boldsymbol{b}_{\mathrm{obs}}, \boldsymbol{b}_{\mathrm{CIB}}\right\rangle}{\left\langle\boldsymbol{b}_{\mathrm{CIB}}, \boldsymbol{b}_{\mathrm{CIB}}\right\rangle}$,

then Eq. (33) can be rewritten to define the joint NG estimators - noted with upper hat - as

$\left(\begin{array}{c}\hat{f}_{\mathrm{NL}} \\ \hat{b}_{\mathrm{PS}} \\ \hat{A}_{\mathrm{CIB}}\end{array}\right)=\mathcal{M}^{-1} \cdot\left(\begin{array}{c}\tilde{f}_{\mathrm{NL}} \\ \tilde{b}_{\mathrm{PS}} \\ \tilde{A}_{\mathrm{CIB}}\end{array}\right)$

with

$\mathcal{M}=\left(\begin{array}{ccc}1 & \Delta f_{\mathrm{NL}}^{\text {unclust }} & \Delta f_{\mathrm{NL}}^{\mathrm{CIB}} \\ \Delta b_{\mathrm{PS}}^{\mathrm{CMB}} & 1 & \Delta b_{\mathrm{PS}}^{\mathrm{ClB}} \\ \Delta A_{\mathrm{CIB}}^{\mathrm{CMB}} & \Delta A_{\mathrm{CIB}}^{\text {unclust }} & 1\end{array}\right)$

and

$$
\begin{array}{cr}
\Delta f_{\mathrm{NL}}^{\text {unclust }}=\frac{\left\langle\boldsymbol{b}_{\text {unclust }}, \boldsymbol{b}_{\mathrm{CMB}}\right\rangle}{\left\langle\boldsymbol{b}_{\mathrm{CMB}}, \boldsymbol{b}_{\mathrm{CMB}}\right\rangle} & \Delta f_{\mathrm{NL}}^{\mathrm{CIB}}=\frac{\left\langle\boldsymbol{b}_{\mathrm{CIB}}, \boldsymbol{b}_{\mathrm{CMB}}\right\rangle}{\left\langle\boldsymbol{b}_{\mathrm{CMB}}, \boldsymbol{b}_{\mathrm{CMB}}\right\rangle} \\
\Delta b_{\mathrm{PS}}^{\mathrm{CMB}}=\frac{\left\langle\boldsymbol{b}_{\mathrm{CMB}}, \boldsymbol{b}_{\text {unclust }}\right\rangle}{\left\langle\boldsymbol{b}_{\text {unclust }}, \boldsymbol{b}_{\text {unclust }}\right\rangle} & \Delta b_{\mathrm{PS}}^{\mathrm{IR}}=\frac{\left\langle\boldsymbol{b}_{\mathrm{CIB}}, \boldsymbol{b}_{\text {unclust }}\right\rangle}{\left\langle\boldsymbol{b}_{\text {unclust }}, \boldsymbol{b}_{\text {unclust }}\right\rangle} \\
\Delta A_{\mathrm{CIB}}^{\mathrm{CMB}}=\frac{\left\langle\boldsymbol{b}_{\mathrm{CMB}}, \boldsymbol{b}_{\mathrm{CIB}}\right\rangle}{\left\langle\boldsymbol{b}_{\mathrm{CIB}}, \boldsymbol{b}_{\mathrm{CIB}}\right\rangle} & \Delta A_{\mathrm{CIB}}^{\text {unclust }}=\frac{\left\langle\boldsymbol{b}_{\mathrm{unclust}}, \boldsymbol{b}_{\mathrm{CIB}}\right\rangle}{\left\langle\boldsymbol{b}_{\mathrm{CIB}}, \boldsymbol{b}_{\mathrm{CIB}}\right\rangle} .
\end{array}
$$

We note in particular that the estimators $\tilde{f}_{\mathrm{NL}}$ and $\tilde{b}_{\mathrm{PS}}$ are the ones defined in Komatsu et al. (2005), while $\tilde{A}_{\mathrm{CIB}}$ is the estimator proposed in the present work.

We compute, for illustration, the mixing matrix at $220 \mathrm{GHz}$. Neglecting the noise and beam effect and assuming the ERCSC flux cuts (Planck Collaboration VII 2011), we find for $\ell_{\max }=$ 2048,

$$
\mathcal{M}=\left(\begin{array}{ccc}
1 & 0.124 & 0.631 \\
2.08 \times 10^{-5} & 1 & 1.79 \\
3.16 \times 10^{-5} & 0.535 & 1
\end{array}\right)
$$

where we renormalised the unclustered sources amplitude to get numbers of order 1 ; i.e., the mixing matrix must be understood to be applied to $b_{\mathrm{PS}} / b_{\mathrm{PS}}^{\text {expected }}$, where $b_{\mathrm{PS}}^{\text {expected }}=1.8 \times 10^{-28}$ is the expected unclustered sources bispectrum that we derived from
Table 3. Correlation coefficients between the different bispectrum templates at $220 \mathrm{GHz}$ with $\ell_{\max }=2048,5$ arcmin Gaussian beam, and $10^{-8} \Delta T / T$ noise.

\begin{tabular}{cccc}
\hline \hline & CIB/unclust & CIB/CMB & unclust/CMB \\
\hline$r$ & $96 \%$ & $0.57 \%$ & $0.24 \%$ \\
\hline
\end{tabular}

the simulations of Sehgal et al. (2010) masking all sources above the ERCSC flux cut $\left(S_{\text {cut }}=0.28 \mathrm{Jy}\right)$.

The matrix is mostly bloc diagonal, with the $f_{\mathrm{NL}}$ part decoupling from the $b_{\mathrm{PS}}$ and $A_{\mathrm{CIB}}$ parts. We have checked that unless $b_{\mathrm{PS}} \gg 10^{-27}, f_{\mathrm{NL}}$ estimation can be considered unaffected by extragalactic foregrounds at this frequency. Conversely, estimation of NG from unclustered and clustered sources is unaffected by $f_{\mathrm{NL}}$. However, we see that the two contributions affect each other heavily, with the $b_{\mathrm{PS}}-A_{\mathrm{CIB}}$ submatrix being poorly conditioned (condition number $\sim 180$ ).

When we include instrumental effects, Table 2, (same $\ell_{\max }$ and flux cut), we find the mixing matrix:

$$
\mathcal{M}=\left(\begin{array}{ccc}
1 & 0.0461 & 0.245 \\
1.21 \times 10^{-4} & 1 & 2.12 \\
1.31 \times 10^{-4} & 0.435 & 1
\end{array}\right)
$$

The relative importance of CMB NG compared to point source NG has increased. Indeed, beam and noise effects mostly decrease the $\mathrm{S} / \mathrm{N}$ in configurations with high multipoles. This down-weights NG from point sources which is dominant at high $\ell$, while CMB NG dominates at lower multipoles. However $\mathrm{CMB}$ and point source NG estimation can still be considered decoupled unless $b_{\mathrm{PS}} \gg 10^{-27}$. In the $b_{\mathrm{PS}}-A_{\mathrm{CIB}}$ submatrix, we see that the relative importance of $b_{\mathrm{PS}}$ has decreased compared to $A_{\mathrm{CIB}}$. Indeed the CIB signal is most important on large angular scales, while the unclustered sources $\mathrm{S} / \mathrm{N}$ comes mostly from small angular scales that are affected by the beam smoothing and the noise.

The $b_{\mathrm{PS}}-A_{\mathrm{CIB}}$ submatrix is still ill-conditioned when we account for beam and noise effect, and this is due to a high correlation between the CIB and unclustered sources' bispectra templates ${ }^{3}$. This correlation can be quantified by the quantity $r=\cos \theta \in[-1,1]$ :

$r_{\alpha, \beta}=\frac{\left\langle\boldsymbol{b}_{\alpha}, \boldsymbol{b}_{\beta}\right\rangle}{\sqrt{\left\langle\boldsymbol{b}_{\alpha}, \boldsymbol{b}_{\alpha}\right\rangle\left\langle\boldsymbol{b}_{\beta}, \boldsymbol{b}_{\beta}\right\rangle}}$

with $\alpha, \beta=$ unclust, $\mathrm{CIB}$, or CMB. In other terms $\theta_{\alpha, \beta}$ is the angle between the vectors $\boldsymbol{b}_{\alpha}, \boldsymbol{b}_{\beta}$ in the vector space of bispectra with the scalar product defined in Eq. (32). At $\ell_{\max }=2048$, with the instrumental configuration of Table 2 , the correlation coefficients of the different bispectra are listed in Table 3.

In Fig. 1, we plot these coefficients as a function of the maximum multipole used in the analysis, with the same instrumental specifications.

When analysing only large angular scales (small $\ell_{\max }$ ), the unclustered and CIB bispectrum templates are anti-correlated with the CMB bispectrum. This is because both unclustered and CIB bispectra estimators are positive, while the CMB is negative due to the Sachs-Wolfe effect term (Bucher et al. 2010). However, when going to higher resolution, the first acoustic peak

3 This correlation of templates has nothing to do with physical correlation of the sources; the populations are still considered independent here. 

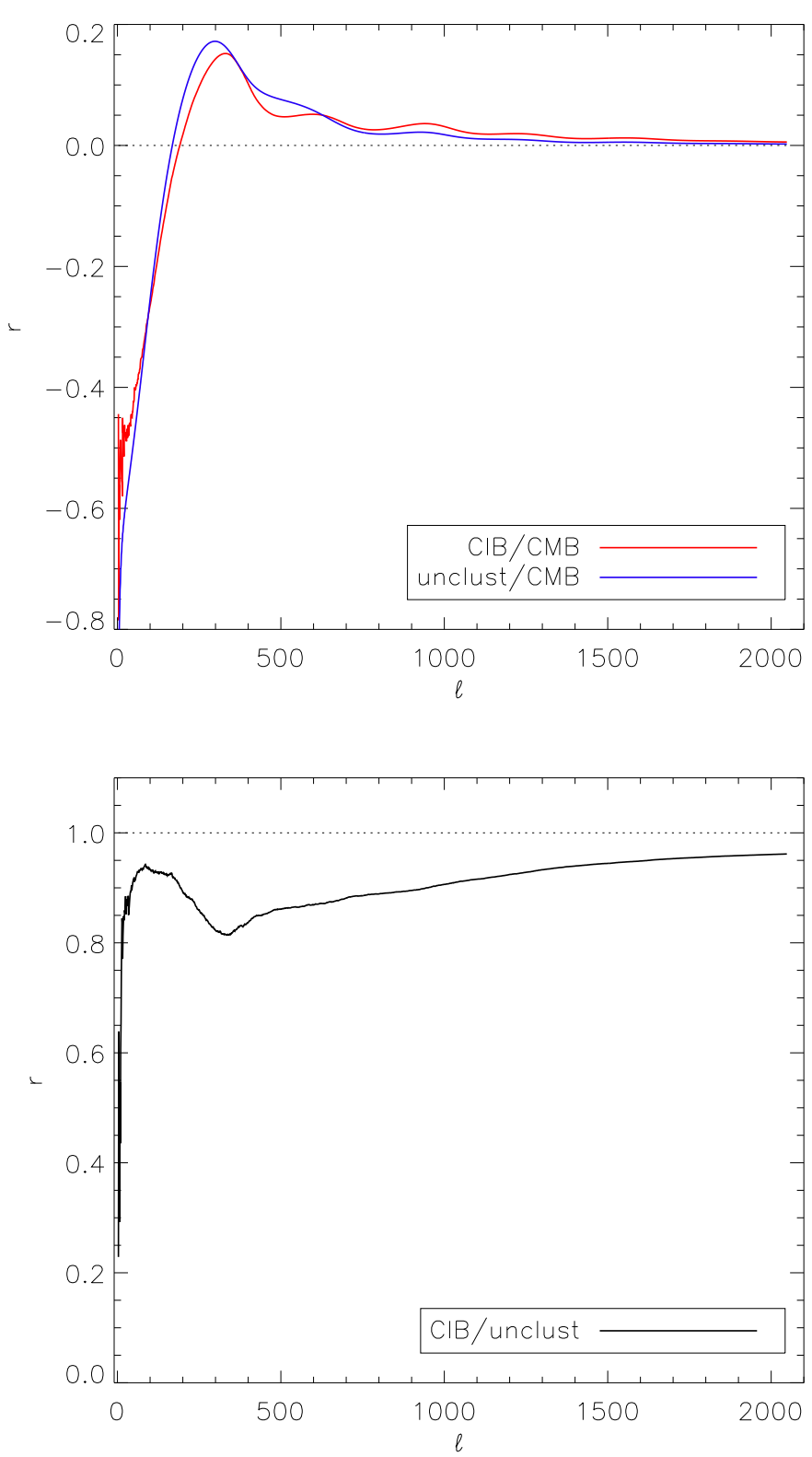

Fig. 1. Correlation coefficients between the radio, CIB, and CMB bispectra at $220 \mathrm{GHz}$ with 5 arcmin Gaussian beam and $10^{-8} \Delta T / T$ noise. Upper panel: correlation between IR and CMB in red, between radio and $\mathrm{CMB}$ in blue, as a function of $\ell_{\max }$. Lower panel: correlation between IR and radio.

makes the CMB bispectrum positive with high values in some configurations (e.g. equilateral). This makes the correlation between the unclustered and CMB bispectrum positive for $\ell \geq 168$. The $\mathrm{CIB} / \mathrm{CMB}$ correlation in turn becomes positive at slightly higher multipoles, $\ell=190$, because the CIB bispectrum peaks in the squeezed configurations where the CMB bispectrum is still negative. At larger multipoles, because of the complex pattern of the CMB bispectrum and the changes of sign with acoustic peaks, the correlation coefficients tend asymptotically to zero.

The CIB and unclustered sources bispectrum templates are positively correlated since they are both positive, and this correlation is weak when analysing only the lowest multipoles, but it increases rapidly over $80 \%$ with a small "dip" at $\ell_{\max }=$ 200-300 at the location of the first CMB acoustic peak. Indeed the CMB spectrum is involved in the denominator of Eq. (32), so at $\ell_{\max }=200-300$ it typically down-weights non-squeezed

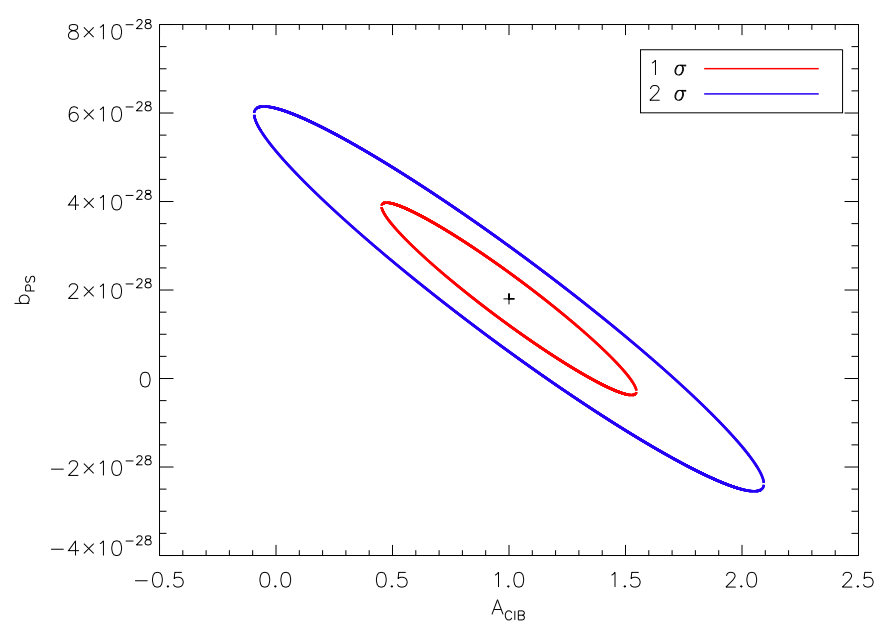

Fig. 2. $1 \sigma$ and $2 \sigma$ confidence levels for $\left(A_{\mathrm{CIB}}, b_{\mathrm{PS}}\right)$ in a joint estimation at $220 \mathrm{GHz} \ell_{\max }=2048$ with a 5 arcmin Gaussian beam and $10^{-8} \Delta T / T$ noise.

configurations where unclustered sources and CIB bispectra are the most correlated. Nevertheless, the correlation between unclustered sources and CIB bispectra asymptotically tends to one at high angular resolutions, at the same time as the CIB bispectrum flattens at high multipoles.

Finally, in Fig. 2 we forecast the likelihood contours in the $\left(A_{\mathrm{CIB}}, b_{\mathrm{PS}}\right)$ plane, which we expect with the aforementioned instrumental specifications. The $A_{\mathrm{CIB}}$ and $b_{\mathrm{PS}}$ estimations are quite degenerate, as expected from the high correlations of their templates, which significantly degrades the constraint that can be put on each of them independently. However, we can still have an order-of-magnitude estimation for both of them. Also, including the prior $b_{\mathrm{PS}} \geq 0$ (and also to some extent $A_{\mathrm{CIB}} \geq 0$ ) would improve the constraints. Finally, CMB removal through component separation may also help by decreasing the variance of both estimators (although not their correlation).

\section{Conclusions}

Statistical methods of estimating the non-Gaussianity from unclustered extragalactic point sources have been developed in the past (Komatsu et al. 2005; Argüeso et al. 2006); however, the subject has not been tackled previously for the case of clustered sources. The prescription proposed in Lacasa et al. (2012) yields a separable form for the bispectrum of clustered sources. This allowed us to build a fast estimator for the clustered sources NG amplitude in this article, à-la-KSW (Komatsu et al. 2005). The estimator can account for a lensed population of sources if the number counts and power spectrum entering as ingredients account for it. We showed how this estimator can be optimised to account for partial sky coverage and inhomogeneous noise. We computed the detection significance that can be expected at several high frequencies dominated by the CIB. Finally we showed how this new estimator can be used with estimators of the unclustered sources and CMB non-Gaussianity to build a joint estimation of sources of non-Gaussianity. We find that the estimation of CMB NG is barely correlated with the estimation of point source NG, so that unless the latter is too important e.g. if few sources are masked- both estimations can be considered decoupled. In particular, this is the case for the cosmological channels (100-220 GHz) with flux cuts lower or equal to that of the Planck Early Release Compact Source Catalog. 
On the contrary, for point sources, clustered and unclustered NG have similar shapes especially at high multipoles that make their amplitude estimation degenerate. We show how this hampers the detectability of the CIB NG at $220 \mathrm{GHz}$. Increasing this detectability can be achieved for example by subtracting the $\mathrm{CMB}$ or by including priors on $b_{\mathrm{PS}}$ in the analysis (e.g. positivity and/or upper limits given by the flux cut and radio-source models).

This study opens up the possibility of robust measurements of non-Gaussianity where contaminations are treated in a systematic way. It also opens up the possibility of detecting point source NG, which may allow their respective models to be constrained.

Acknowledgements. The authors thank an anonymous referee for the comments and suggestions. They thank B. Wandelt for interesting discussions. They acknowledge the use of the HEALpix package (Gorski et al. 2005).

\section{References}

Acquaviva, V., Bartolo, N., Matarrese, S., \& Riotto, A. 2003, Nucl. Phys. B, 667, 119

Argüeso, F., González-Nuevo, J., \& Toffolatti, L. 2003, ApJ, 598, 86

Argüeso, F., Sanz, J. L., Barreiro, R. B., Herranz, D., \& González-Nuevo, J. 2006, MNRAS, 373, 311

Babich, D., \& Pierpaoli, E. 2008, Phys. Rev. D, 77, 123011

Béthermin, M., Daddi, E., Magdis, G., et al. 2012, ApJ, 757, L23

Bobin, J., Starck, J.-L., Sureau, F., \& Basak, S. 2013, A\&A, 550, A73

Bucher, M., Tent, B. V., \& Carvalho, C. S. 2010, MNRAS, 407, 2193

Crawford, T. M., Schaffer, K. K., Bhattacharya, S., et al. 2014, ApJ, 784, 143

Creminelli, P. \& Zaldarriaga, M. 2004, J. Cosmol. Astropart. Phys., 2004, 006
Creminelli, P., Nicolis, A., Senatore, L., Tegmark, M., \& Zaldarriaga, M. 2006, J. Cosmol. Astropart. Phys., 2006, 004

Curto, A., Tucci, M., González-Nuevo, J., et al. 2013, MNRAS, 432, 728

Das, S., Marriage, T. A., Ade, P. A. R., et al. 2011, ApJ, 729, 62

Delabrouille, J., \& Cardoso, J. 2007 [arXiv: astro-ph/0702198]

Elsner, F., \& Wandelt, B. D. 2013, A\&A, 549, A111

González-Nuevo, J., Toffolatti, L., \& Argüeso, F. 2005, ApJ, 621, 1

Gorski, K. M., Hivon, E., Banday, A. J., et al. 2005, ApJ, 622, 759

Hall, N. R., Keisler, R., Knox, L., et al. 2010, ApJ, 718, 632

Keisler, R., Reichardt, C. L., Aird, K. A., et al. 2011, ApJ, 743, 28

Komatsu, E., Spergel, D. N., \& Wandelt, B. D. 2005, ApJ, 634, 14

Komatsu, E., Dunkley, J., Nolta, M. R., et al. 2009, ApJS, 180, 330

Komatsu, E., Smith, K. M., Dunkley, J., et al. 2011, ApJS, 192, 18

Lacasa, F., Aghanim, N., Kunz, M., \& Frommert, M. 2012, MNRAS, 421, 1982

Lapi, A., González-Nuevo, J., Fan, L., et al. 2011, ApJ, 742, 24

Maldacena, J. 2003, J. High Energy Phys., 2003, 013

Pénin, A., Doré, O., Lagache, G., \& Béthermin, M. 2012, A\&A, 537, A137

Planck Collaboration I. 2011, A\&A, 536, A1

Planck Collaboration VII. 2011, A\&A, 536, A7

Planck Collaboration XVIII. 2011, A\&A, 536, A18

Planck Collaboration I. 2014, A\&A, in press, DOI: 10.1051/0004-6361/201321529

Planck Collaboration XXIV. 2014, A\&A, in press, DOI: 10.1051/0004-6361/201321554

Planck Collaboration XXX. 2014, A\&A, in press, DOI: $10.1051 / 0004-6361 / 201322093$

Remazeilles, M., Delabrouille, J., \& Cardoso, J.-F. 2011, MNRAS, 410, 2481

Sehgal, N., Bode, P., Das, S., et al. 2010, ApJ, 709, 920

Somerville, R. S., Gilmore, R. C., Primack, J. R., \& Domínguez, A. 2012, MNRAS, 423, 1992

Thacker, C., Cooray, A., Smidt, J., et al. 2013, ApJ, 768, 58

Toffolatti, L., Argueso Gomez, F., de Zotti, G., et al. 1998, MNRAS, 297, 117

Tucci, M., Toffolatti, L., De Zotti, G., \& Martínez-González, E. 2011, A\&A, 533, A57

Viero, M. P., Wang, L., Zemcov, M., et al. 2013, ApJ, 772, 77

Xia, J.-Q., Negrello, M., Lapi, A., et al. 2012, MNRAS, 422, 1324 